"This is the peer reviewed version of the following article: Liu, X., He, Y., Zhang, J., and Zeng, G. (2017) A new state-of-charge estimation method for electric vehicle lithium-ion batteries based on multiple input parameter fitting model. Int. J. Energy Res.[Accepted Author Manuscript], which has been published in final form at [http://dx.doi.org/10.1002/er.3705] This article may be used for non-commercial purposes in accordance with Wiley Terms and Conditions for Self-Archiving." 


\title{
A New State-of-Charge Estimation Method for Electric Vehicle Lithium-Ion Batteries based on Multiple Input Parameter Fitting Model
}

\author{
Xintian LIU, Yao HE, Xinxin ZHENG, Jiangfeng ZHANG, Guojian ZENG
}

New Energy Automobile Engineering Research Institute, Hefei University of Technology, Anhui, China

\begin{abstract}
The estimation of State of Charge (SOC) is crucial
\end{abstract} to determine the remaining capacity of the Lithium-Ion battery, and thus plays an important role in many electric vehicle control and energy storage system management problems. The accuracy of the estimated SOC mostly depends on the accuracy of the battery model, which is mainly affected by factors like temperature, State of Health and chemical reactions. Also the SOC can reflect the output characteristics of the battery cell. The battery model is often identified by experiments under different SOCs and temperatures, which represent a large amount of work. To resolve this difficulty and also improve modeling accuracy, a multiple input parameter fitting model of the Lithium-Ion battery and the factors which would affect the accuracy of the battery model are derived from the Nernst equation in this paper. Statistics theory is applied to obtain a more accurate battery model while using less measurement data. The relevant parameters can be fitted by multiple factors, including continuously changing temperature. From the obtained battery model, Extended Kalman Filter algorithm is applied to estimate the SOC. Finally, simulation and experimental results are given to prove the advantage of the proposed SOC estimation method. It is found that the proposed SOC estimation method always satisfies the precision requirement in the relevant Standards under different environmental temperatures while existing traditional methods cannot. Particularly, the SOC estimation accuracy can be improved by $14 \%$ under low temperatures below $0^{\circ} \mathrm{C}$ compared to existing methods.

Index Terms-Nernst Equation, State of Charge, Battery Model, Design of Experiment, Extended Kalman Filter

\section{INTRODUCTION}

Being the main power source of electric vehicles, Lithium-Ion battery remains a heated research topic for many years. The concept State-of-Charge (SOC) refers to the remaining capacity of the battery, and is one of the most important objects to study Lithium-Ion batteries [1]. SOC needs to be estimated by the Battery Management System (BMS) in order to achieve proper controls for electric vehicles [2], for instance, SOC is indispensable for the driving distance forecast, charging mode selection, and many other demand side management applications $[3,4]$. An error in the estimation of the SOC will threaten the safety and reliability of the battery pack [5]. Therefore, the accurate estimation of SOC is an important target of BMS, and this paper aims to solve this accuracy problem and provide a novel SOC estimation method for Lithium-Ion batteries.

The ampere-hour integral method is a common SOC estimation method, where the change of the SOC is characterized by the integral of the current over a given time period $[6,7]$. The ampere-hour integral method is indeed an open-loop estimation method, therefore, current sampling error and system noise often reduce the accuracy of this SOC estimation method. Moreover, the error of the estimated SOC can also be accumulated over time and can deviate much from true values. In addition, the initial value of the SOC is difficult to determine $[8,10]$. To solve these problems, advanced filtering algorithms can be applied in the SOC estimation, where feedback techniques in these algorithms can improve estimation tolerance to accumulated errors. This SOC estimation technique can be further integrated into the battery model. Theoretical output voltage calculated from the new battery model will be treated as a reference value, while the actual output voltage can be measured and treated as a feedback to the battery 
model. The difference between the reference and the feedback is regulated and a compensation value is introduced to compensate the SOC estimation result. As a result, the SOC estimation can achieve very high accuracy.

The accuracy of SOC estimation relies on an accurate battery model. If the battery model is not accurate enough, then the advanced filtering algorithms have only limited improvement on the accuracy of SOC estimation. Usually there are two types of battery models, the equivalent circuit model and the electrochemistry model. The equivalent circuit model uses a DC voltage source, a resistor and a capacitor to describe the Lithium-Ion battery [11]. Typical examples of equivalent circuit models include the Thevenin model and the PNGV model introduced in [12] and [13]. The structures of these models are different, and the parameters of these models are difficult to identify. An electrochemistry model is based on the chemical reaction inside the battery [14]. For this type of model, the relationship between system parameters is clear, and the parameters are easy to identify. Most of the existing electrochemistry models are based on the Nernst equation [15]-[17]. However, the influence of the temperature is rarely taken into consideration, and there is a need to introduce a temperature compensation coefficient introduced to improve the accuracy of the battery model. In addition, to identify the battery system parameters, a large number of experiments need to be performed, and the inclusion of the temperature parameter will increase the work load exponentially.

There are existing studies on advanced filtering algorithms for the improvement of battery model accuracy. For instance, a neural network filtering algorithm is proposed in [18] to realise real-time estimation. However, this method requires a large quantity of training data. Then the Extended Kalman Filtering algorithm (EKF) is proposed in [19]-[20] and the Unscented Particle Filtering algorithm is presented in [21], and both algorithms can achieve high accuracy. A novel online estimation technique for estimating the SOC is also shown in [22], where a fading Kalman filter is used to estimate the open circuit voltage from the battery model parameters. Generally, EKF algorithm can achieve accurate SOC estimation and is easy to realize, therefore, it is widely applied in the SOC estimation.

From the above analysis, this paper will propose a novel SOC estimation method based on the Nernst equation and EKF algorithm. It can achieve high accuracy without large amount of measurement data, which can effectively reduce experimental workload. The obtained battery model has multiple input factors, which can capture information from continuously changing environment. The Design of Experiment (DOE) based on the statistical theory is applied to fit the internal resistance of the battery [23]-[24]. As a result, the model has the advantages of high accuracy and small identification workload. Based on the multiple input parameter fitting model, the EKF is applied further to estimate the SOC, which can inhibit the initial SOC and estimation errors. The experimental and simulation results show that compared to the traditional EKF algorithm based on a normal Nernst battery model, in the low temperature below $0^{\circ} \mathrm{C}$, with the proposed SOC estimation method, the estimation accuracy can be improved by $14 \%$. In the normal temperature, the estimation accuracy can be improved by $7 \%$.

This paper is organised as follows. Section II introduces the electrochemistry model based on the Nernst equation and analyses the factors that affect the change of parameters. An improved method of the Nernst model is given. In Section III, the principle of DOE is discussed, and the internal resistance fitting method is proposed. In Section IV, the other parameters of the Lithium-Ion battery model, such as the standard electrode potential and the logarithmic term coefficient are identified. The influence of the temperature is also taken into consideration. In Section V, the EKF method based on the battery model is applied to estimate the SOC. The accuracy of the proposed method is demonstrated by the simulation and experiment in Section VI, which is followed by concluding remarks in Section VII.

\section{MUlTIPLE INPUT BATTERY MODEL}

Nernst equation is an electrochemical expression of the battery electromotive force based on the reversible chemical reaction. The Nernst equation can be expressed as

$$
E=E_{0}-\frac{R T_{\mathrm{K}}}{n F} \ln \frac{\alpha_{\mathrm{red}}}{\alpha_{\text {ox }}}
$$

where $T_{\mathrm{k}}$ is the thermodynamics temperature of chemical reaction; $E$ is the electrode potential under $T_{\mathrm{k}}$, and it is also the open circuit voltage of the battery; $E_{0}$ is the standard electrode potential, which can be observed as the open circuit voltage when the battery is fully charged; $R$ is the gas constant $8.314 \mathrm{~J} \cdot \mathrm{K}^{-1} \cdot \mathrm{mol}^{-1} ; F$ is the Faraday constant and equals $96485 \mathrm{C} / \mathrm{mol} ; n$ is the number of the electrons that participate in the electrode reaction; $\alpha_{\mathrm{ox}}$ and $\alpha_{\text {red }}$ are the ion activities of the high and low oxidised side, respectively [15]. The oxidation-reduction reaction of the $\mathrm{Li}^{+}$is expressed as 


$$
\mathrm{Li}^{+}+e^{-} \Leftrightarrow \mathrm{Li}
$$

It can be observed that $n$ is 1 , thus, (1) can be rewritten as

$$
E=E_{0}+\frac{R T_{\mathrm{K}}}{F} \ln \frac{\alpha_{\mathrm{Li}^{+}}}{\alpha_{\mathrm{Li}}}
$$

$\mathrm{Li}$ is in the solid state, so $\alpha_{\mathrm{Li}}$ is 1 [25], and $\alpha_{\mathrm{Li}+}$ is

$$
\alpha_{\mathrm{Li}^{+}}=c_{\mathrm{Li}^{+}} \gamma_{\mathrm{Li}^{+}}
$$

where $c_{\mathrm{Li}+}$ is the concentration, and $\gamma_{\mathrm{Li}+}$ is the activity coefficient of the $\mathrm{Li}^{+}$. The quantitative relationship between $c_{\mathrm{Li}+}$ and $\gamma_{\mathrm{Li}+}$ is difficult to identify, but generally the higher $c_{\mathrm{Li}+}$ is, the lower $\gamma_{\mathrm{Li}+}$ is. The concentration $c_{\mathrm{Li}+}$ can reflect the uncharged capacity of the battery, that is, the higher $c_{\mathrm{Li}}$ is, the greater the uncharged capacity of the battery is. Thus a higher $\gamma_{\mathrm{Li}}$ implies a larger residual capacity of the battery. The remaining capacity of the battery is represented by SOC, therefore the uncharged capacity of the battery can be expressed as (1-SOC). Then $c_{\mathrm{Li}+}$ and $\gamma_{\mathrm{Li}+}$ are positively correlated to (1-SOC) and SOC, respectively, that is, they are in proportional positive correlations or exponential positive correlations. Thus (4) can be simplified as one of the following two forms:

$$
\begin{gathered}
\alpha_{\mathrm{Li}^{+}}=\beta_{1}(1-\mathrm{SOC}) \beta_{2} \mathrm{SOC} \\
\alpha_{\mathrm{Li}^{+}}=(1-\mathrm{SOC})^{\beta_{1}} \mathrm{SOC}^{\beta_{2}}
\end{gathered}
$$

where $\beta_{1}$ and $\beta_{2}$ are constants to be identified and represent the impact of 1-SOC and SOC, respectively. In (5), the location of $\beta_{1}$ and $\beta_{2}$ can exchange with each other. So this equation cannot reflect the pertinence of the parameters and must be discarded. Therefore, equation (6) is selected to represent (4).

There is no quantitative relationship between $c_{\mathrm{Li}+}$ and 1-SOC or between $\gamma_{\mathrm{Li}+}$ and SOC. As a result, the coefficients $\beta_{1}$ and $\beta_{2}$ can be fitted via experimental data. According to (3) and (6), the output voltage of the battery $U$ is

$$
U=E-I_{c} R_{i n}=E_{0}-I_{c} R_{i n}+k_{1} \ln (1-\mathrm{SOC})+k_{2} \ln \mathrm{SOC}
$$

where $I_{\mathrm{c}}$ is the current of the battery, $R_{\text {in }}$ is the internal resistance, $k_{1}$ and $k_{2}$ are the simplified coefficient, which can be expressed as follows:

$$
\left\{\begin{array}{l}
k_{1}=R \beta_{1} T_{K} / F=R \beta_{1}\left(T_{\mathrm{C}}+273\right) / F \\
k_{2}=R \beta_{2} T_{K} / F=R \beta_{2}\left(T_{\mathrm{C}}+273\right) / F
\end{array}\right.
$$

$T_{\mathrm{C}}$ is the temperature in centigrade. $k_{1}$ and $k_{2}$ have a relationship with $T_{\mathrm{C}}$. Further research should be performed to identify these relationships.

In (7), the internal resistance $R$ in is not a constant, as it is affected by the SOC and the State of Health (SOH) [26]. The temperature $T_{\mathrm{C}}$ also has an influence on the value of $R_{\text {in. }}$. A mathematical model will be established for $R_{\text {in }}$ in the next section.

\section{MODELING OF THE INTERNAL RESISTANCE}

\section{A. Principle of the design of experiment}

To analyse the relationship among $R_{\text {in }}, \mathrm{SOC}, \mathrm{SOH}$ and $T_{\mathrm{C}}$, a large number of experiments is necessary to obtain a sufficient amount of data. The Hybrid Pulse Power Characteristic (HPPC) method is applied to measure the internal resistance under given values of SOC, $\mathrm{SOH}$ and $T_{\mathrm{C}}$. It will require a large amount of time to measure the internal resistance under different values of these SOC, SOH and $T_{\mathrm{c}}$. Since different types of Lithium-Ion batteries have different internal resistances; the obtained internal resistance model will usually be applicable to specific types of battery only. To solve this problem, DOE is applied to establish the internal resistance model with as low amount of data as possible [27]. In this way, the modelling period can be shortened. This DOE based modelling method is universal for different types of Lithium-Ion batteries. Actually, DOE is widely applied in aerospace industry, manufacturing industry and medical science. DOE is a modelling method that originated in statistical theory, with multiple inputs and multiple outputs [28-29]. Central Composite Design (CCD) is a widely used specific type of DOE method with particularly simple system designs [30]. Therefore, CCD is applied in this paper to establish the internal resistance model.

The DOE internal resistance model based on the CCD is shown in Fig. 1. The inputs to this model are $\mathrm{SOC}, \mathrm{SOH}$ and $T_{\mathrm{C}}$. The output is $R_{\text {in. }}$

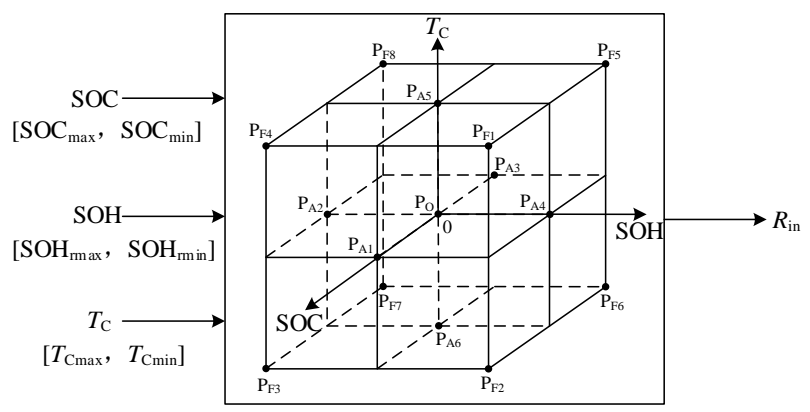

Fig. 1. The DOE internal resistance model based on the CCD The central point $\mathrm{P}_{\mathrm{O}}$ in Fig. 1 is in the origin of coordinate. 
There are $2^{3}$ factorial points and $2 \times 3$ axial points. The factorial points are $\mathrm{P}_{\mathrm{F} 1} \sim \mathrm{P}_{\mathrm{F} 8}$, and the axial points are $\mathrm{P}_{\mathrm{A} 1} \sim \mathrm{P}_{\mathrm{A} 6}$.

According to statistics theory, the model can be treated as a quadratic surface if the parameter range is sufficiently small [31]. In the selected range, if the residuals of the fitting and actual value obey normal distribution and limit in the 6 standard deviation $(6 \sigma)$, then the model is accurate [32]. In this paper, the internal resistance of each point is measured, and the approximated quadratic surface can be fitted. If the residuals of the calculated and the measured values of $R_{\text {in }}$ satisfy the $6 \sigma$ normal distribution, then the quadratic surface can be treated as the model of the internal resistance.

\section{B. $\quad$ Modelling method based on DOE}

Relevant terms of the internal resistance model are shown in Table 1.

Table 1. The terms of the internal resistance model.

\begin{tabular}{ccc}
\hline Type & Term & Coefficient \\
\hline Quadratic Term & $\mathrm{SOC}^{2}$ & $a_{1}$ \\
& $\mathrm{SOH}^{2}$ & $a_{2}$ \\
& $T_{\mathrm{C}}{ }^{2}$ & $a_{3}$ \\
\hline Coupling Term & $\mathrm{SOC} \times \mathrm{SOH}$ & $b_{1}$ \\
& $\mathrm{SOC} \times T_{\mathrm{C}}$ & $b_{2}$ \\
& $\mathrm{SOH} \times T_{\mathrm{C}}$ & $b_{3}$ \\
\hline Linear Term & $\mathrm{SOC}$ & $c_{1}$ \\
& $\mathrm{SOH}$ & $c_{2}$ \\
\hline Constant Term & $T_{\mathrm{C}}$ & $c_{3}$ \\
\hline
\end{tabular}

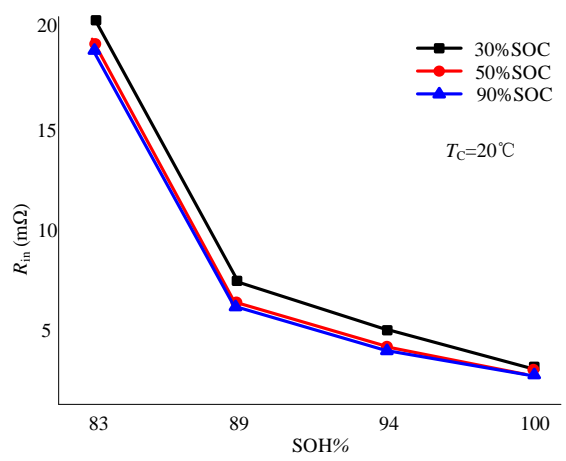

(a)

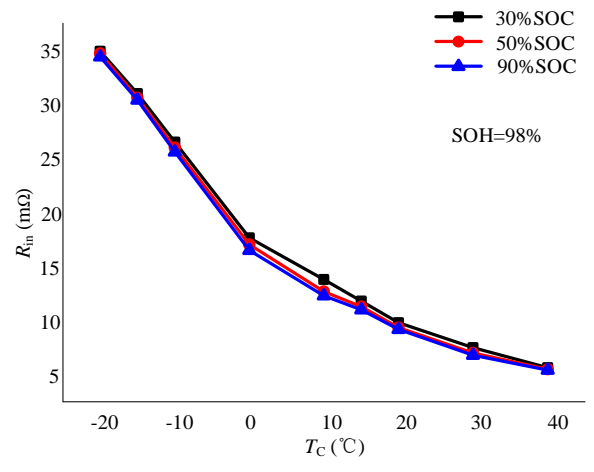

(b)

Fig. 2. The relationship among $R_{\text {in }}, \mathrm{SOC}, \mathrm{SOH}$ and $T_{\mathrm{C}}$ (a) The relationship between $R_{\text {in }}$ and SOH under different SOC (b) The relationship between $R_{\text {in }}$ and $T_{\mathrm{C}}$ under different SOC

The internal resistance $R$ in can be measured by the Hybrid Pulse Power Characteristic (HPPC) method. Fig. 2 shows the relationship among $R_{\text {in }}, \mathrm{SOC}, \mathrm{SOH}$ and $T_{\mathrm{C}}$. It can be observed that three inputs all have influences on $R_{\text {in }}$. For any given SOC, $R_{\text {in }}$ increases with the increment of $T_{\mathrm{C}}$. In contrast, $R_{\text {in }}$ decreases with the increment of SOH.

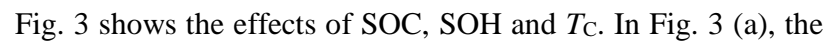
effect of $T_{\mathrm{C}}$ alone is the most obvious, and is higher than that of SOC and SOH. In Fig. 3 (b), two of these three inputs are chosen to analyse the interactive effect, while the third input is set to be different values. There are six types of combination of the three inputs, namely, the relationship between the SOC and $R_{\text {in }}$ with different $T_{\mathrm{c}}$, relationship between the $\mathrm{SOH}$ and $R_{\text {in }}$ with different $T_{\mathrm{c}}$, relationship between $T_{\mathrm{c}}$ and $R_{\text {in }}$ with different SOC, relationship between $\mathrm{SOH}$ and $R_{\text {in }}$ with different SOC, relationship between $T_{\mathrm{c}}$ and $R_{\text {in }}$ with different $\mathrm{SOH}$, and relationship between SOC and $R_{\text {in }}$ with different $\mathrm{SOH}$.

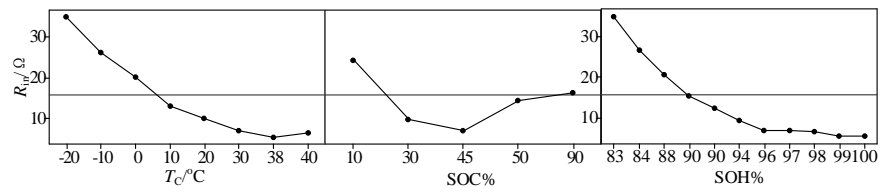

(a) 


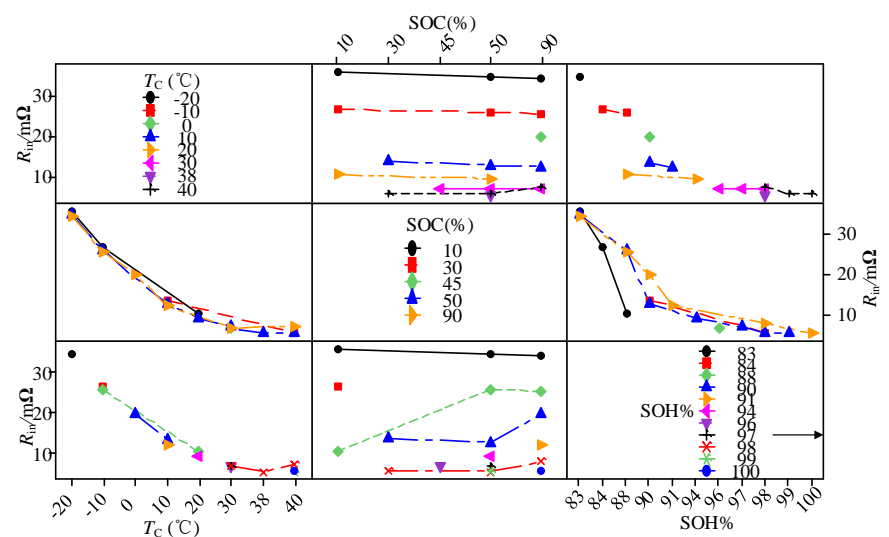

(b)

Fig. 3. Effects of SOC and $T$ to $R_{\text {in. }}$ (a) Impact of a single input (b) Interaction effect

If the lines are almost parallel in Fig 3 (b), then the interaction effect of the two corresponding inputs is not obvious. Therefore, the coefficients of the coupling terms are small. For example, the lines in the interaction effect figure of SOC and $T_{\mathrm{C}}$ are almost parallel. Thus, the coefficient $b_{2}$ in Table 1 is small. And the lines in the interaction effect figure of $T_{\mathrm{C}}$ and $\mathrm{SOH}$ are not regular, to be fitted with experimental data [33].

\section{IDENTIFICATION OF THE OTHER PARAMETERS}

\section{A. Identification of the open circuit voltage}

In the existing Nernst model, $E_{0}, R_{\text {in }}, k_{1}$, and $k_{2}$ are the fixed values. In fact, these parameters are changing with the environment. The calculation of $R_{\text {in }}$ is discussed above. The identification of the other parameters require further analysis.

Fig. 4 shows the measured $E_{0}$ distribution under different environmental temperature; it can be well approximated as the following linear equation:

$$
E_{0}\left(T_{C}\right)=\gamma_{1} T_{C}+\gamma_{2}
$$

where $\gamma_{1}$ and $\gamma_{2}$ are constant coefficients. According to Fig. 7, $\gamma_{1}$ and $\gamma_{2}$ are 0.006 and 3.3346, respectively.

Table 2. The values of key points in Fig 1

\begin{tabular}{|c|c|c|c|}
\hline Type & $\mathrm{SOC} / \%$ & $\mathrm{SOH} / \%$ & $T_{\mathrm{C} /}{ }^{\circ} \mathrm{C}$ \\
\hline Central Point & $\left(\mathrm{SOC}_{\min }+\mathrm{SOC}_{\max }\right) / 2$ & $\left(\mathrm{SOH}_{\min }+\mathrm{SOH}_{\max }\right) / 2$ & $\left(T_{\mathrm{C} \min }+T_{\mathrm{Cmax}}\right) / 2$ \\
\hline \multirow[t]{8}{*}{ Factorial Point } & $\mathrm{SOC}_{\min }$ & $\mathrm{SOH}_{\min }$ & $T_{\mathrm{Cmin}}$ \\
\hline & $\mathrm{SOC}_{\min }$ & $\mathrm{SOH}_{\max }$ & $T_{\text {Cmin }}$ \\
\hline & $\mathrm{SOC}_{\min }$ & $\mathrm{SOH}_{\min }$ & $T_{\text {Cmax }}$ \\
\hline & $\mathrm{SOC}_{\min }$ & $\mathrm{SOH}_{\max }$ & $T_{\mathrm{Cmax}}$ \\
\hline & $\mathrm{SOC}_{\max }$ & $\mathrm{SOH}_{\text {min }}$ & $T_{\text {Cmin }}$ \\
\hline & $\mathrm{SOC}_{\max }$ & $\mathrm{SOH}_{\max }$ & $T_{\text {Cmin }}$ \\
\hline & $\mathrm{SOC}_{\max }$ & $\mathrm{SOH}_{\text {min }}$ & $T_{\text {Cmax }}$ \\
\hline & $\mathrm{SOC}_{\max }$ & $\mathrm{SOH}_{\max }$ & $T_{\mathrm{Cmax}}$ \\
\hline \multirow[t]{6}{*}{ Axial Point } & $\mathrm{SOC}_{\min }$ & $\left(\mathrm{SOH}_{\min }+\mathrm{SOH}_{\max }\right) / 2$ & $\left(T_{\mathrm{Cmin}}+T_{\mathrm{Cmax}}\right) / 2$ \\
\hline & $\mathrm{SOC}_{\max }$ & $\left(\mathrm{SOH}_{\min }+\mathrm{SOH}_{\max }\right) / 2$ & $\left(T_{\mathrm{C} \min }+T_{\mathrm{Cmax}}\right) / 2$ \\
\hline & $\left(\mathrm{SOC}_{\min }+\mathrm{SOC}_{\text {max }}\right) / 2$ & $\mathrm{SOH}_{\min }$ & $\left(T_{\mathrm{C} \min }+T_{\mathrm{Cmax}}\right) / 2$ \\
\hline & $\left(\mathrm{SOC}_{\min }+\mathrm{SOC}_{\text {max }}\right) / 2$ & $\mathrm{SOH}_{\max }$ & $\left(T_{\mathrm{C} \min }+T_{\mathrm{C} \max }\right) / 2$ \\
\hline & $\left(\mathrm{SOC}_{\min }+\mathrm{SOC}_{\max }\right) / 2$ & $\left(\mathrm{SOH}_{\min }+\mathrm{SOH}_{\max }\right) / 2$ & $T_{\text {Cmin }}$ \\
\hline & $\left(\mathrm{SOC}_{\min }+\mathrm{SOC}_{\text {max }}\right) / 2$ & $\left(\mathrm{SOH}_{\min }+\mathrm{SOH}_{\max }\right) / 2$ & $T_{\text {Cmax }}$ \\
\hline
\end{tabular}

which implies the coefficient $b_{3}$ in Table 1 is large.

Table 2 shows the values of key points described in Fig. 1. The corresponding internal resistances related to these points need to be tested through experiments. The quadratic internal resistance is easy 


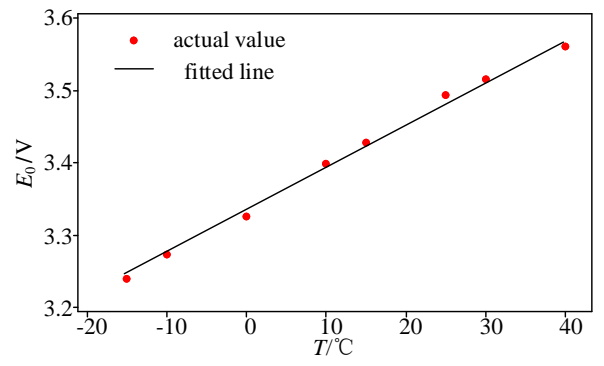

Fig. 4. Relationship between $E_{0}$ and $T$

\section{B. Identification of $k_{1}$ and $k_{2}$}

The open circuit voltage of the battery $E$ can be tested under different temperatures and SOCs. At any given temperature, $k_{1}$ and $k_{2}$ in (8) can be fitted using the least square method. For instance, denote the SOC by $x$ and E by $y$, then the regression model can be expressed as

$$
f(x)=k_{1} \ln (1-x)+k_{2} \ln x
$$

The quadratic loss function is given as

$$
Q=\sum_{j=1}^{m}[y-f(x)]^{2}
$$

where $m$ is the number of the measurement points. The over-determined matrix $R_{\mathrm{M}}$ is

$$
R_{M}=\left[\begin{array}{cc}
\ln \left(1-x_{1}\right) & \ln \left(x_{1}\right) \\
\ln \left(1-x_{2}\right) & \ln \left(x_{2}\right) \\
\cdots & \cdots \\
\ln \left(1-x_{m}\right) & \ln \left(x_{m}\right)
\end{array}\right]
$$

The fitted values of $k_{1}$ and $k_{2}$ can be written as

$$
\left[\begin{array}{l}
k_{1} \\
k_{2}
\end{array}\right]=\left(R_{M}{ }^{T} R_{M}\right)^{-1} R_{M}\left[\begin{array}{c}
y_{1} \\
y_{2} \\
\cdots \\
y_{m}
\end{array}\right]
$$

Table 3 shows the values of $k_{1}$ and $k_{2}$ under different temperatures, where RMSE represents the Root-Mean-Square-Error. The very small values of RMSE prove the accuracy of the fitted results.

Table 3 Parameter identification

\begin{tabular}{cccc}
\hline$T_{\mathrm{C}}$ & $k_{1}$ & $k_{2}$ & RMSE \\
\hline$-15^{\circ} \mathrm{C}$ & 0.06806 & 0.02293 & 0.01519 \\
$-10^{\circ} \mathrm{C}$ & 0.07346 & 0.02873 & 0.01214 \\
$10^{\circ} \mathrm{C}$ & 0.1037 & 0.03391 & 0.02018 \\
$15^{\circ} \mathrm{C}$ & 0.131 & 0.04636 & 0.0249 \\
$30^{\circ} \mathrm{C}$ & 0.2014 & 0.0801 & 0.03313 \\
\hline
\end{tabular}

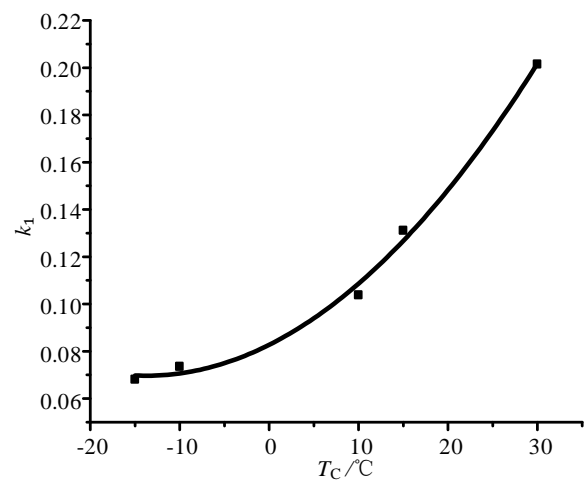

(a)

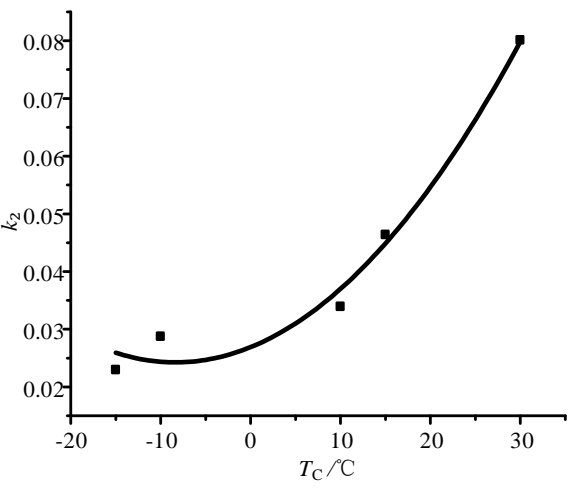

(b)

Fig. 5. Dependence on $T_{\mathrm{C}}$ of $k_{1}$ and $k_{2}$ (a) Relationship between $k_{1}$ and $T_{\mathrm{C}}(\mathrm{b})$ Relationship between $k_{2}$ and $T_{\mathrm{C}}$

Fig. 5 shows the relationship between $k_{1}, k_{2}$ and $T_{\mathrm{C}}$ with

$$
k_{i}=\eta_{i 1} T^{2}+\eta_{i 2} T+\eta_{i 3}(i=1,2)
$$

The coefficients $\eta_{\mathrm{i} 1}, \eta_{\mathrm{i} 2}$ and $\eta_{\mathrm{i} 3}$ are shown in Table 4 .

Table 4 Coefficients of $k_{1}$ and $k_{2}$

\begin{tabular}{ccc}
\hline$i$ & 1 & 2 \\
\hline$\eta_{\mathrm{i} 1}$ & $6.89558 \mathrm{e}-5$ & $3.78042 \mathrm{e}-5$ \\
$\eta_{\mathrm{i} 2}$ & 0.0019 & $6.3134 \mathrm{e}-4$ \\
$\eta_{\mathrm{i} 3}$ & 0.08275 & 0.0269 \\
\hline
\end{tabular}

\section{SOC ESTIMATION BASED ON EKF ALGORITHM}

The SOC can be described by the following equation:

$$
\operatorname{SOC}(t)=\operatorname{SOC}(0)-\int_{0}^{t} \frac{i(t) d t}{Q_{\mathrm{r}}}
$$

where is $Q_{\mathrm{r}}$ the actual capacity of the battery. Thus, the discrete state-space battery model can be written as

$$
\left\{\begin{array}{l}
x_{k+1}=x_{k}-\frac{\Delta t}{Q_{r}} i_{k} \\
y_{k}=E_{0}-R_{i n} i_{k}+k_{1} \ln \left(1-x_{k}\right)+k_{2} \ln x_{k}+v_{k}
\end{array}\right.
$$


where $v_{\mathrm{k}}$ is the observation noise of voltage measurement. The state transition matrix $\boldsymbol{A}$ and the observation matrix $\boldsymbol{C}$ are defined as

$$
\left\{\begin{array}{l}
A_{k-1}=\left.\frac{\partial f\left(x_{k-1}, i_{k-1}\right)}{\partial x_{k-1}}\right|_{x_{k-1}=\hat{x}_{k-1}}=1 \\
C_{k}=\left.\frac{\partial g\left(x_{k}, i_{k}\right)}{\partial x_{k}}\right|_{x_{k}=\hat{x}}=-\frac{k_{1}}{1-x_{k}}+\frac{k_{2}}{x_{k}}
\end{array}\right.
$$

where the function $f(x, i)$ and $g(x, i)$ are the observation equation (see (7)) and state equation (see (15)), respectively. The steps of EKF algorithm are as follows:

(i) Initialise the variables for $k=0$.

(ii) Update the iteration index $k$ by $k+1$ each time. The iteration equations of the state and error covariance are determined by:

$$
\left\{\begin{array}{l}
\hat{x}_{k}^{-}=f\left(\hat{x}_{k-1}, i_{k-1}\right) \\
P_{k}^{-}=A_{k} P_{k-1} A_{k}^{T}+Q
\end{array}\right.
$$

(iii) The Kalman gain can be calculated by the following equation:

$$
K_{k}=P_{k}^{-} C_{k}^{T}\left(C_{k} P_{k}^{-} C_{k}^{T}+R\right)^{-1}
$$

$\boldsymbol{Q}$ and $\boldsymbol{R}$ is the error covariance matrix of $f(x, i)$ and $g(x, i)$, respectively.

(iv) The observation iterative equations of the state and error covariance can be calculated according to the following equations:

$$
\left\{\begin{array}{l}
\hat{x}_{k}=\hat{x}_{k}^{-}+K_{k}\left[y_{k}-g\left(\hat{x}_{k}^{-}, i_{k}\right)\right] \\
P_{k}=\left(I-K_{k} C_{k}\right) P_{k}^{-}
\end{array}\right.
$$

After performing iterative calculations, the effects of system noise can be effectively filtered and the accuracy of the battery SOC estimation can be significantly improved.

\section{EXPERIMENTAL AND SIMULATION RESULTS}

To verify the accuracy of the newly proposed SOC estimation method in the previous sections, Lithium battery experiments are performed. The rated capacity of the Lithium battery is $9 \mathrm{Ah}$, and the experimental steps are as follows:

(i) The Lithium battery is fully charged and then discharged to $90 \%$ SOC.

(ii) The battery is placed in a thermostat for $30 \mathrm{~min}$. Next, a 10-s peak discharge experiment is performed. The discharge current is $4 \mathrm{C}$.

(iii) A constant current discharge experiment is performed. The discharging current is $0.5 \mathrm{C}$. This process does not stop until $20 \%$ capacity of the battery is discharged.

(iv) The battery is let stand for $30 \mathrm{~min}$. Next, repeat steps (ii) to (iv) for another 3 times.

Fig. 6(a) shows the experimental device. The experiment is done in the environmental chamber, which can realize continuously changing temperatures. Fig. 6(b) shows the current waveforms of the experiment. The top and bottom waveforms are the discharging current and current noise, respectively.

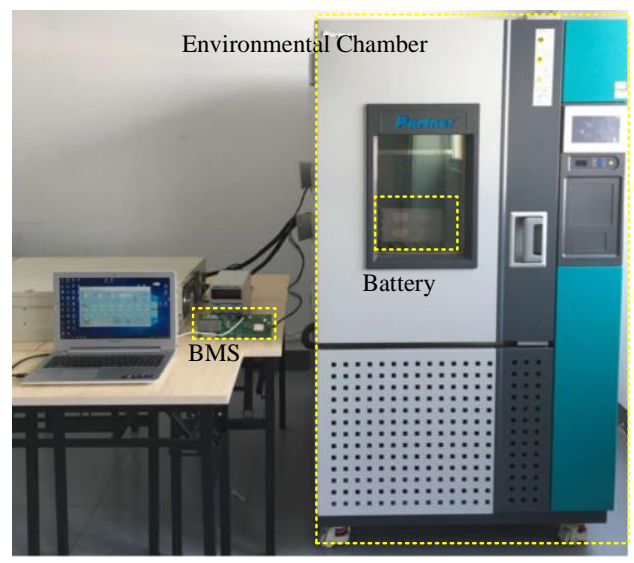

(a)

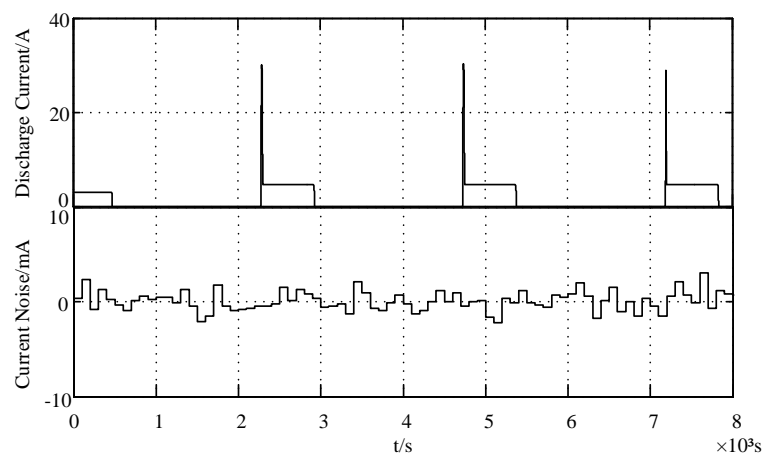

(b)

Fig. 6. The experimental condition (a) The experimental device (b) The current waveforms of the experiment

Table 5 shows the experimental data, which provides measured values for Table 2. The internal resistance model can be established according to these data. The fitted coefficients are shown in Table 6.

Table 5. Experimental data

\begin{tabular}{ccccc}
\hline Type & $\mathrm{SOC} / \%$ & $\mathrm{SOH} / \%$ & $T_{\mathrm{C}} /{ }^{\circ} \mathrm{C}$ & $R_{\text {in }} / \mathrm{m} \Omega$ \\
\hline Central Point & 60 & 92 & 10 & 12.5 \\
\hline Factorial Point & 30 & 83 & -20 & 34.9 \\
& 30 & 100 & -20 & 31 \\
\hline
\end{tabular}




\begin{tabular}{ccccc}
\hline & 30 & 83 & 40 & 18.4 \\
& 30 & 100 & 40 & 5.56 \\
& 90 & 83 & -20 & 34.4 \\
& 90 & 100 & -20 & 31.2 \\
& 90 & 83 & 40 & 6.32 \\
& 90 & 100 & 40 & 5.43 \\
\hline Axial Point & 30 & 92 & 10 & 13.8 \\
& 90 & 92 & 10 & 12.3 \\
& 60 & 83 & 10 & 19.7 \\
& 60 & 100 & 10 & 5.92 \\
& 60 & 92 & -20 & 26 \\
& 60 & 92 & 40 & 7.35 \\
\hline
\end{tabular}

Table 6. The fitted coefficients

\begin{tabular}{ccccc}
\hline$a_{1}$ & $a_{2}$ & $a_{3}$ & $b_{1}$ & $b_{2}$ \\
\hline $7.56 \mathrm{e}-4$ & $-6.21 \mathrm{e}-2$ & $9.13 \mathrm{e}-3$ & $-3.211 \mathrm{e}-4$ & $6.73 . \mathrm{e}-4$ \\
\hline$b_{3}$ & $c_{1}$ & $c_{2}$ & $c_{3}$ & $C_{\text {on }}$ \\
\hline $1.07 \mathrm{e}-2$ & $-5.82 \mathrm{e}-2$ & 10.97 & -1.605 & -463.12 \\
\hline
\end{tabular}

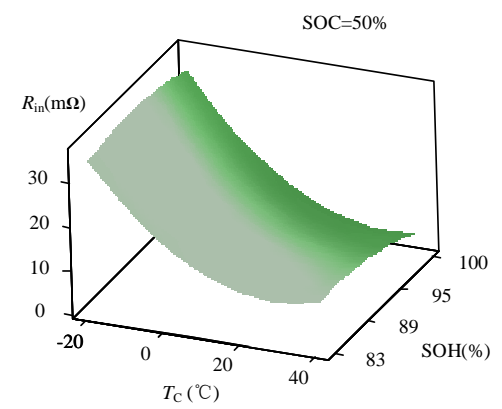

(a)

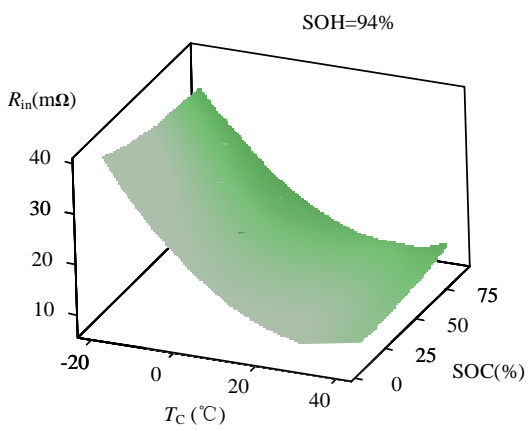

(b)

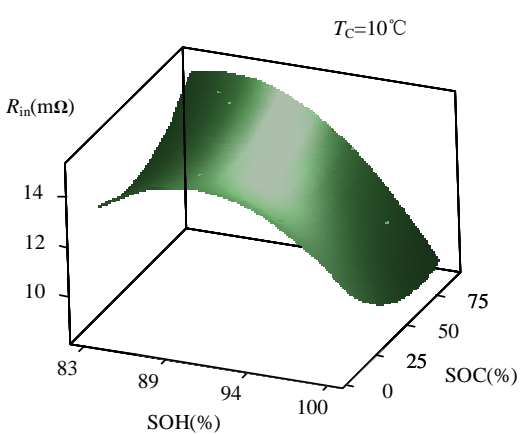

(c)

Fig. 7. The quadric surface of $R_{\text {in }}$ (a) $\mathrm{SOC}$ is set as $50 \%$ (b) $\mathrm{SOH}$ is set as $94 \%$ (c) $T_{\mathrm{C}}$ is set as $10^{\circ} \mathrm{C}$

Fig. 7 shows the quadric surface of $R_{\text {in }}$, and Fig. 8 shows the corresponding contour map. The influence of both the temperature $T_{\mathrm{c}}$ and actual capacity on $\mathrm{SOH}$ are observed.

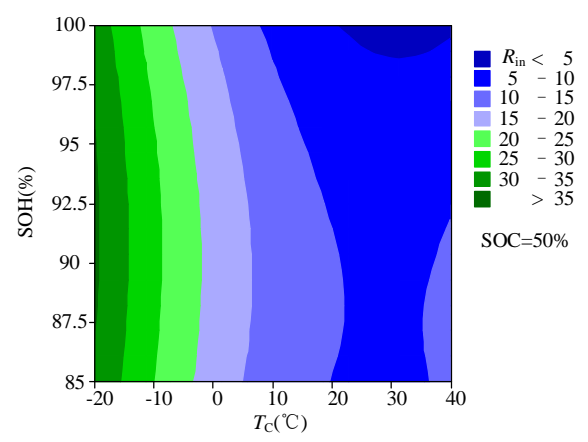

(a)

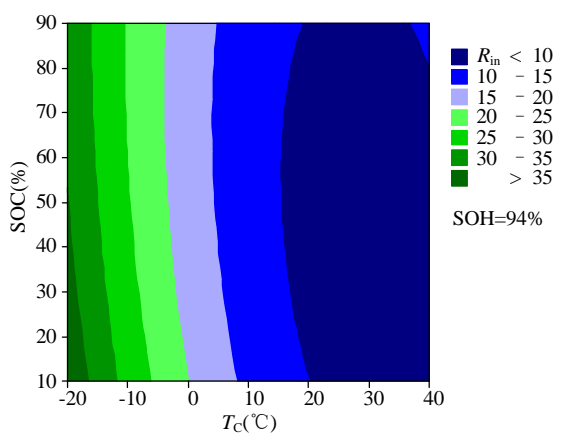

(b)

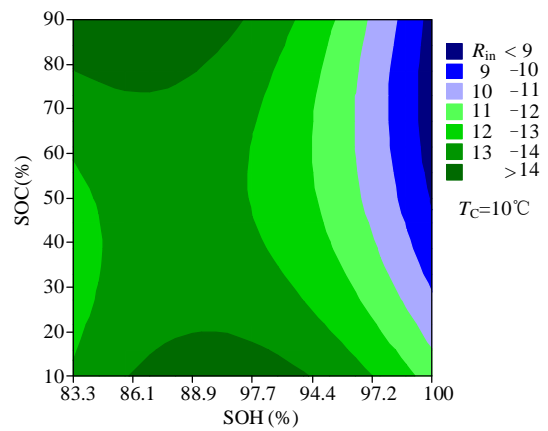

(c)

Fig. 8. The contour map of $R_{\text {in }}$ (a) SOC is set as $50 \%$ (b) SOH is set as $94 \%$ (c) $T_{\mathrm{C}}$ is set as $10^{\circ} \mathrm{C}$ 


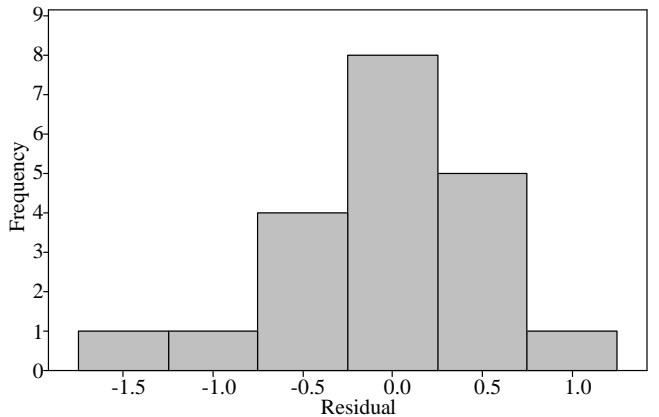

Fig. 9. Residual histogram of $R$ in

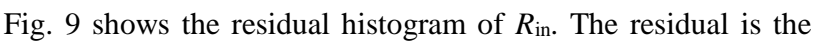
difference between the observed value and the fitted value. The residual value follows approximately a normal distribution, indicating that the internal resistance calculated in the model is applicable to capture continuous changes of the three inputs $T \mathrm{c}$, $\mathrm{SOH}$ and SOC.

From the experimental results, a Lithium battery model is established with the aid of MATLAB/Simulink. In the experiment, the tested temperatures for parameter identification are $-20^{\circ} \mathrm{C}$, $40^{\circ} \mathrm{C}$ and $10^{\circ} \mathrm{C}$, respectively. For Lithium vehicle batteries, the ambient temperature ranges from $-20^{\circ} \mathrm{C}$ to $40^{\circ} \mathrm{C}$. In order to prove the accuracy of the battery model, some other temperature points need to be chosen for test. In the experiment, the temperature testing points are set as $-15^{\circ} \mathrm{C}, 15^{\circ} \mathrm{C}$ and $30^{\circ} \mathrm{C}$. In addition, a classical Nernst model of the Lithium battery is established. The parameters of the model are tested under the condition that Tc is $25^{\circ} \mathrm{C}$ and $\mathrm{SOH}$ is 1 . The classic Nernst model is shown as follows: $U=3.49-0.008 I_{c}+0.187 \ln (1-\mathrm{SOC})+0.0723 \ln \mathrm{SOC}(21)$

Fig. 10 shows the SOC curves. $\mathrm{EKF}_{1}$ is the estimated value of SOC obtained by proposed estimation method. $\mathrm{EKF}_{2}$ is the estimated value of SOC based on the classical Nernst model. Fig. 10 (a) shows the SOC curves under the temperature $-15^{\circ} \mathrm{C}$. The three curves are the real SOC, the SOC estimated by EKF 1 and $\mathrm{EKF}_{2}$, respectively It can be seen that the SOC estimated by $\mathrm{EKF}_{1}$ is closer to the real SOC than that estimated by $\mathrm{EKF}_{2}$. Fig. 10 (b) and (c) show the SOC curves under the temperatures $15^{\circ} \mathrm{C}$ and $30^{\circ} \mathrm{C}$. Also the SOC estimated by $\mathrm{EKF}_{1}$ is closer to the real SOC than that estimated by $\mathrm{EKF}_{2}$.

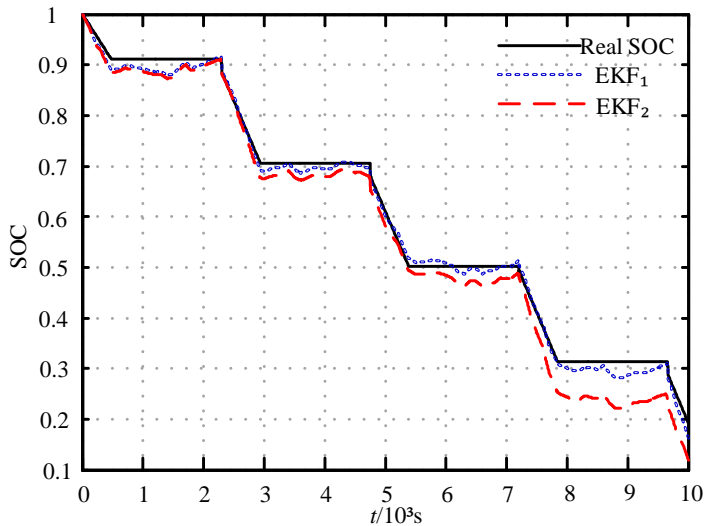

(a)

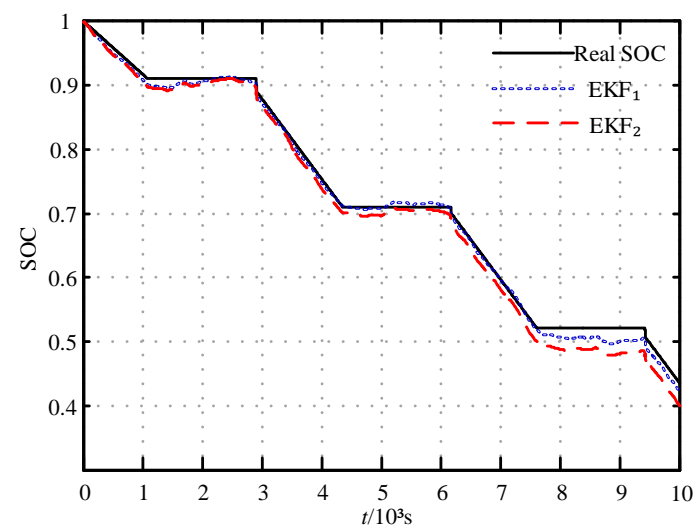

(b)

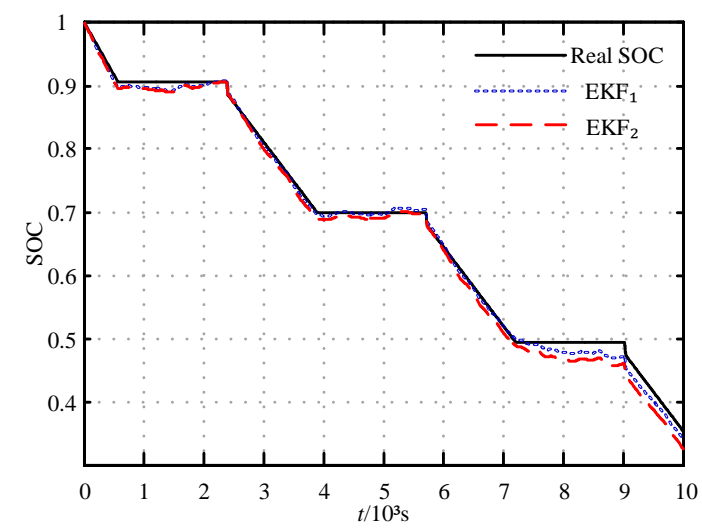

(c)

Fig. 10. SOC curves (a) $T_{\mathrm{C}}=-15^{\circ} \mathrm{C}$ (b) $T_{\mathrm{C}}=15^{\circ} \mathrm{C}$ (e) $T_{\mathrm{C}}=30^{\circ} \mathrm{C}$

Fig. 11 shows the error curves which correspond to Fig. 10. The error curves show the error of the real SOC and the estimated SOC. The error can be calculated as follows:

$$
\text { error }=\frac{\mathrm{SOC}_{\text {real }}-\mathrm{SOC}_{\text {estimation }}}{\mathrm{SOC}_{\text {real }}}
$$

Fig. 11 (a), (b) and (c) show the error curves under the temperature $-15^{\circ} \mathrm{C}, 15^{\circ} \mathrm{C}$ and $30^{\circ} \mathrm{C}$. The comparison of the three figures shows that in the low temperature environment, the error 
of the real SOC and the estimated SOC is higher than that in the normal temperature environment. Because the parameters of the battery model change obviously after the temperature decreases below $0^{\circ} \mathrm{C}$. The accuracy of low temperature SOC estimation is low. With the proposed method, the accuracy can be improved.

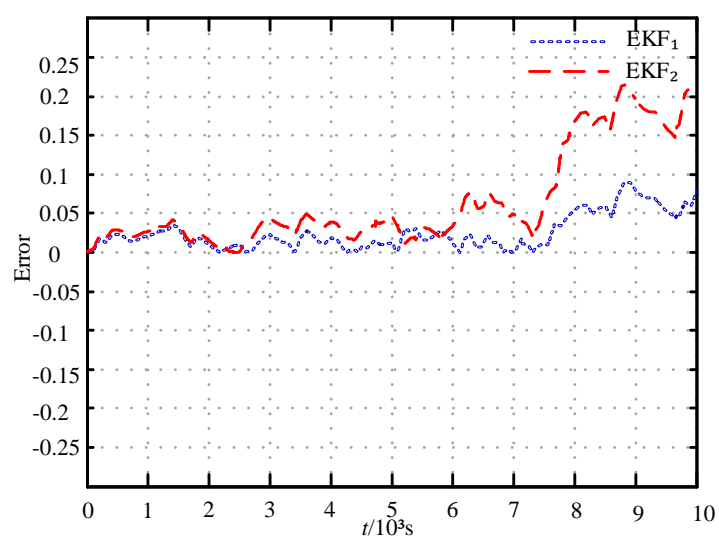

(a)

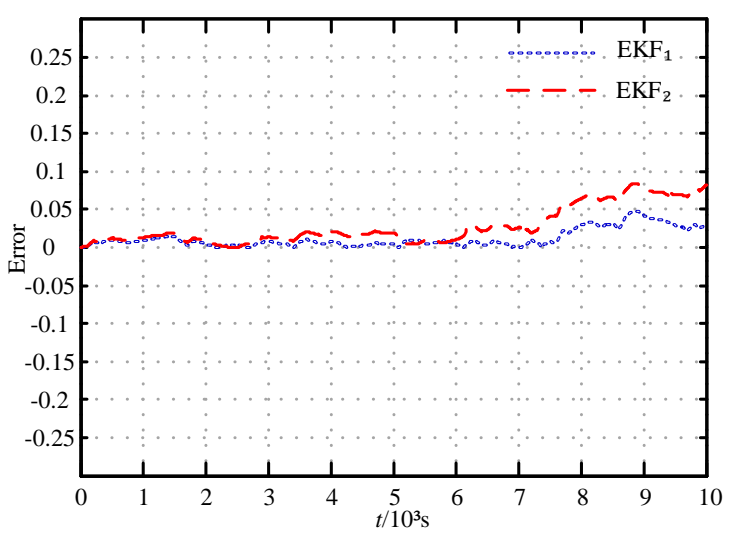

(b)

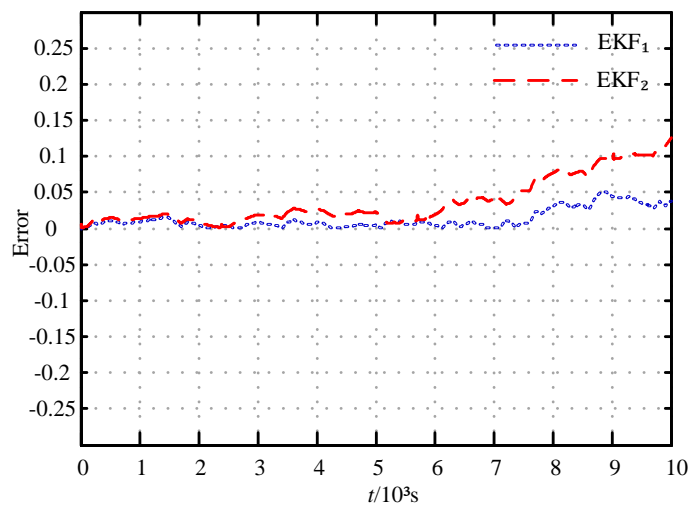

(c)

Fig. 11. Error curves (a) $T_{\mathrm{C}}=-15^{\circ} \mathrm{C}$ (b) $T_{\mathrm{C}}=15^{\circ} \mathrm{C}$ (e) $T_{\mathrm{C}}=30^{\circ} \mathrm{C}$
The experimental time at $-15^{\circ} \mathrm{C}$ is shorter than that of $15^{\circ} \mathrm{C}$ and $30^{\circ} \mathrm{C}$ because the real capacity of the battery decreases with the decrease of temperature. As a result, a shorter time is required to release the same proportion of capacity at low temperatures.

According to the Automobile Industry Standards of China QCT 897-2011, Section 4.2.4 [34], the maximum allowable error of SOC estimation between temperatures $5^{\circ} \mathrm{C} \sim 15^{\circ} \mathrm{C}$ or $25^{\circ} \mathrm{C} \sim 35^{\circ} \mathrm{C}$ must not exceed $10 \%$. It can be seen from Fig. 11 (a) that in the low temperature environment, $\mathrm{EKF}_{2}$ cannot meet this $10 \%$ accuracy requirement in the Standards; however, this requirement is always met by $\mathrm{EKF}_{1}$. Details of the maximum relative errors of the SOC estimation methods under different temperatures are show in Table 7.

Table 7. Maximum relative errors

\begin{tabular}{cccc}
\hline Temperature & $\mathrm{EKF}_{1}$ & $\mathrm{EKF}_{2}$ & Improved Percentage \\
\hline$-15^{\circ} \mathrm{C}$ & 0.09 & 0.23 & 0.14 \\
$15^{\circ} \mathrm{C}$ & 0.05 & 0.08 & 0.03 \\
$30^{\circ} \mathrm{C}$ & 0.05 & 0.12 & 0.07 \\
\hline
\end{tabular}

Table 7 convinces that precision of the proposed SOC estimation method always satisfies the $10 \%$ accuracy requirement in the Standards under different environmental temperatures. In particular, this new method can improve $14 \%$ accuracy at low temperatures compared to traditional Nernst method.

\section{CONCLUSIONS}

In this paper, a novel modelling method based on the Nernst equation has been proposed. It has multiple input factors, which can satisfy continuously changing environment. Statistical theory is applied to obtain a more accurate battery model while using less measurement data. The DOE method has been applied to achieve high accuracy of the battery model and reduce experiment workload. Also the accuracy of the DOE model is easy to validate when the residuals of the fitting and actual value obey normal distribution and limit in the 6 standard deviation. Based on the multiple input parameter fitting model, EKF algorithm can realize high accuracy SOC estimation. Finally experimental and simulation results are given. Compared to the traditional EKF algorithm based on a normal Nernst battery model, with the proposed SOC estimation method, the estimation accuracy maximally can be improved by $14 \%$. 


\section{REFERENCES}

[1] Osswald P J, Erhard S V, Rheinfeld A, et al. "Temperature dependency of state of charge inhomogeneities and their equalization in cylindrical lithium-ion cells". Journal of Power Sources, 329 (2016): 546-552.

[2] Huang W, Qahouq J A A. "Energy sharing control scheme for state-of-charge balancing of distributed battery energy storage system". IEEE Transactions on Industrial Electronics, 62, no. 5 (2015): 2764-2776

[3] Fotouhi A, Auger D J, Propp K, et al. "A review on electric vehicle battery modelling: From Lithium-ion toward Lithium-Sulphur". Renewable and Sustainable Energy Reviews, 56, (2016): 1008-1021.

[4] Dai, Haifeng, et al. "ANFIS (adaptive neuro-fuzzy inference system) based online SOC (State of Charge) correction considering cell divergence for the EV (electric vehicle) traction batteries." Energy 80 (2015): 350-360.

[5] Marongiu, Andrea, Marco Roscher, and Dirk Uwe Sauer. "Influence of the vehicle-to-grid strategy on the aging behavior of lithium battery electric vehicles." Applied Energy 137 (2015): 899-912.

[6] Zheng, Z. H. U., et al. "SOC Estimation of LiFePO4 Battery based on Improved Ah Integral Method." TELKOMNIKA Indonesian Journal of Electrical Engineering 11.12 (2013): 7058-7064.

[7] Zhang, Cheng, et al. "An integrated approach for real-time model-based state-of-charge estimation of lithium-ion batteries." Journal of Power Sources 283 (2015): 24-36.

[8] Sun F, Xiong R, He H. "A systematic state-of-charge estimation framework for multi-cell battery pack in electric vehicles using bias correction technique”. Applied Energy, 162 (2016): 1399-1409.

[9] Zou Y, Hu X, Ma H, et al. "Combined state of charge and state of health estimation over lithium-ion battery cell cycle lifespan for electric vehicles". Journal of Power Sources, 273 (2015): 793-803.

[10] Zhang L, Hu X, Wang Z, et al. "Fractional-order modeling and State-of-Charge estimation for ultracapacitors". Journal of Power Sources, 314 (2016): 28-34

[11] Greenleaf, Michael, et al. "A Temperature-Dependent Study of Sealed Lead-Acid Batteries Using Physical Equivalent Circuit Modeling With Impedance Spectra Derived High Current/Power Correction." Sustainable Energy, IEEE Transactions on 6.2 (2015): 380-387.

[12] Xiong $\mathrm{R}$, $\mathrm{He} \mathrm{H}$, Zhao K. "Research on an Online Identification Algorithm for a Thevenin Battery Model by an Experimental Approach". International Journal of Green Energy, 12, no. 3 (2015): 272-278.

[13] Leslie, Kenneth, Ilteris Demirkiran, Eric Rask, and Henning Lohse-Busch. "An investigation into the PNGV battery model with the 11 addition of a dynamic temperature range." In Southeastcon, 2013 Proceedings of IEEE, pp. 1-6. IEEE, 2013.

[14] Stetzel, Kirk D., et al. "Electrochemical state and internal variables estimation using a reduced-order physics-based model of a lithium-ion cell and an extended Kalman filter." Journal of Power Sources 278 (2015): 490-505.

[15] Chang, Fengqi, and Zedong Zheng. "An SOC estimation method based on sliding mode observer and the Nernst Equation." In 2015 IEEE Energy Conversion Congress and Exposition (ECCE), pp. 6187-6190. IEEE, 2015.

[16] Ranom, Rahifa, Giles Richardson, and Jamie Michael Foster. "Mathematical Model for Moderately Dilute Electrolytes of Lithium Ion Battery." In Meeting Abstracts, no. 4, pp. 720-720. The Electrochemical Society, 2014.

[17] Liu, Xin-tian, Shao-xun Qin, Yao He, Xin-xin Zheng, and Cheng-rong Cao. "SOC estimation of the lithium-ion battery with the temperature-based Nernst model." In Power Electronics and Motion Control Conference (IPEMC-ECCE Asia), 2016 IEEE 8th International, pp. 1419-1422. IEEE, 2016.

[18] He, Wei, et al. "State of charge estimation for Li-ion batteries using neural network modeling and unscented Kalman filter-based error cancellation." International Journal of Electrical Power \& Energy Systems 62 (2014): 783-791.

[19] Sepasi S, Roose L R, Matsuura M M. "Extended kalman filter with a fuzzy method for accurate battery pack state of charge estimation". Energies, 8, no. 6 (2015): 5217-5233.

[20] Pérez G, Garmendia M, Reynaud J F, et al. "Enhanced closed loop State of Charge estimator for lithium-ion batteries based on Extended Kalman Filter". Applied Energy, 155 (2015): 834-845.

[21] He, Yao, et al. "A new model for State-of-Charge (SOC) estimation for high-power Li-ion batteries." Applied Energy 101 (2013): 808-814.

[22] Lim K C, Bastawrous H A, Duong V H, et al. "Fading Kalman filter-based real-time state of charge estimation in LiFePO 4 battery-powered electric vehicles”. Applied Energy, 169 (2016): 40-48.

[23] Zheng, Xinxin, Lan Xiao, Yun Lei, and Zilong Wang. "Optimisation of LCL filter based on closed-loop total harmonic distortion calculation model of the grid-connected inverter." IET Power Electronics 8, no. 6 (2015): 860-868.

[24] Gu, Bing, and Diane J. Burgess. "Prediction of dexamethasone release from PLGA microspheres prepared with polymer blends using a design of experiment approach." International journal of pharmaceutics 495.1 (2015): 393-403.

[25] Vidal-Iglesias, Francisco J., et al. "Do you really understand the 
electrochemical Nernst equation?." Electrocatalysis 4.1 (2013): 1-9.

[26] Piłatowicz G, Marongiu A, Drillkens J, et al. “A critical overview of definitions and determination techniques of the internal resistance using lithium-ion, lead-acid, nickel metal-hydride batteries and electrochemical double-layer capacitors as examples". Journal of Power Sources, 296 (2015): 365-376.

[27] Shiao, Yao Jung, Mei Ling Jow, Wen Hwa Kuo, Quang Anh Nguyen, and Chao Wei Lai. "Design and Experiment of the Magnetorheological Damper with Multiple Poles." In Applied Mechanics and Materials, vol. 764, pp. 223-227. Trans Tech Publications, 2015.

[28] Sadeghifam, Aidin Nobahar, et al. "Combined use of design of experiment and dynamic building simulation in assessment of energy efficiency in tropical residential buildings." Energy and Buildings 86 (2015): 525-533.

[29] Kumar D, Meenan BJ, Mutreja I, , et al. "Controlling the size and size distribution of gold nanoparticles: a design of experiment study." International Journal of Nanoscience 11.02 (2012): 1250023.

[30] Li Z Z, Xuan D J, Li Y, et al. "Optimization for cooling system of batteries having porous material using design of experiments". International Journal of Precision Engineering and Manufacturing, 16, no. 2 (2015): 275-279.

[31] Myers, Raymond H., Douglas C. Montgomery, and Christine M. Anderson-Cook. Response surface methodology: process and product optimization using designed experiments. John Wiley \& Sons, 2016.

[32] Zhang M, Wang W, Goh T N, et al. "Comprehensive Six Sigma application: a case study". Production Planning \& Control, 26, no. 3 (2015): 219-234.

[33] Candioti L V, De Zan M M, Camara M S, et al. "Experimental design and multiple response optimization. Using the desirability function in analytical methods development". Talanta, 124 (2014): 123-138.

[34] QCT 897-2011, Technical specification battery management system electricvehicles [S]. China, 2011.

Xintian Liu received his $\mathrm{PhD}$ degree in automation from University of Science and Technology of China in 2011. He is the Chief Lecturer for an international cooperation programmer in Anhui Province on power lithium battery lifecycle management system. He was the managing director and chief engineer of Anhui LIGOO New Energy Technology Co., Ltd from 2010 to 2012. He is currently an associate professor at the New Energy Automotive Engineering Research Institute, Hefei University of Technology.
Yao He received the $\mathrm{BSc}$ and $\mathrm{PhD}$ degrees in automation from the University of Science and Technology of China, Hefei, China, in July 2007 and October 2012, respectively. His current research interests include Battery Management System and battery equalization. He is currently an associate professor at the New Energy Automotive Engineering Research Institute, Hefei University of Technology. He was a winner for the Anhui Science and Technology Award for his achievement on new energy automotive power lithium battery technology and key management systems.

Xinxin Zheng received her BSc and $\mathrm{PhD}$ degrees in electrical engineering from Nanjing University of Aeronautics and Astronautics, Nanjing, Jiangsu, in 2009 and 2015, respectively. She is currently working at the New Energy Automobile Engineering Research Institute, Hefei University of Technology. Her research interest is power electronics.

Guojian Zeng received the bachelor's degree in automation from University of Science and Technology of China in 2011. He is currently working at the New Energy Automotive Engineering Research Institute, Hefei University of Technology. His current research interests include Battery Management System, State-of-Power of Li-ion Battery and energy recovery of electric vehicle. 